\title{
Citoyenneté et rituels funéraires des immigrants. Le cas de migrants congolais au Canada
}

Citizenship and Funerals of Immigrants: A Focus on Congolese Immigrants in Canada

Ciudadanía y los rituales funerarios de los inmigrantes. El caso de la República Democrática del Congo

\section{Mambo Tabu Masinda}

\section{(Q) OpenEdition}

\section{Journals}

Édition électronique

URL : https://journals.openedition.org/remi/6997

DOI : $10.4000 /$ remi.6997

ISSN : $1777-5418$

Éditeur

Université de Poitiers

Édition imprimée

Date de publication : 1 décembre 2014

Pagination : 219-230

ISBN : 979-10-90426-23-8

ISSN : 0765-0752

Référence électronique

Mambo Tabu Masinda, « Citoyenneté et rituels funéraires des immigrants. Le cas de migrants

congolais au Canada », Revue européenne des migrations internationales [En ligne], vol. $30-n^{\circ} 3$ et 4 I

2014, mis en ligne le 01 décembre 2017, consulté le 16 avril 2022. URL : http://

journals.openedition.org/remi/6997 ; DOI : https://doi.org/10.4000/remi.6997 


\section{Note de recherche}

\section{Citoyenneté et rituels funéraires des immigrants. Le cas de migrants congolais au Canada}

\section{Mambo Tabu Masinda ${ }^{1}$}

\section{Introduction}

Avec le développement de l'immigration qu'entraîne la globalisation dans toutes les sphères de la vie humaine, les exigences des rituels mortuaires des immigrants se présentent de plus en plus comme une réalité difficile à ignorer (Fall et Dimé, 2011 ; Chaib, 2000 ; Aggoun, 2003 ; Petit, 2002). Mais, malgré un intérêt croissant porté à la question de la mort en migration comme en témoignent des recherches récentes (Kadrouch-Outmany, 2013 ; Van den Breemer et Maussen, 2012 ; Baubock, 2010), celle-ci demeure insuffisamment traitée par les sciences sociales (Lestage, 2012). Cet article a pour objectif de porter un regard sur la citoyenneté des immigrants vue sous l'angle de la justice sociale qu'apporte la reconnaissance de leurs rituels funéraires. Nous souhaitons montrer comment la non-reconnaissance des rituels funéraires des immigrants porte atteinte à leur citoyenneté. La reconnaissance culturelle permet en effet d'assurer à tous les citoyens, quels que soient leurs origines ethniques ou leurs statuts sociaux, la justice sociale dont ils ont besoin afin de se sentir valorisés et intégrés dans les sociétés d'accueil.

Les recherches portant sur les rituels mortuaires des immigrants traitent de plusieurs thématiques parmi lesquelles l'ambivalence quant au lieu d'inhumation du défunt lorsque la mort survient dans le pays d'accueil et l'inadaptation des lieux funéraires aux pratiques funéraires des immigrants (Rachédi, Leduc et Montgomery, 2010). Jusqu'alors, peu d'analyses ont été développées à propos du contenu de la citoyenneté du pays d'accueil en relation avec les pratiques funéraires des immigrants. En d'autres mots, l'immigrant peut-il se sentir un citoyen à part entière d'un État qui lui refuse la possibilité d'exercer les pratiques funéraires dont il est l'héritier? Dans le contexte de la globalisation, les aspects politique, civique et social ne suffisent plus à faire de la citoyenneté un ingrédient humainement valorisant dans la mesure où la citoyenneté moderne

1 Conseiller en adaptation scolaire, Burnaby School District, Unit 79, 8676158 Street,

Surrey, British Columbia, V4N 5W3, Canada ; mambo_masinda@yahoo.ca 
englobe de multiples constellations (Baubock, 2010) parmi lesquelles les droits relatifs aux rituels funéraires. De ce point de vue, l'analyse de la citoyenneté à travers la reconnaissance des rituels funéraires contribue comme nous allons le montrer à enrichir le débat sur la citoyenneté des immigrants.

L'argumentaire s'articule autour d'une brève introduction, suivie d'une revue des connaissances sur les rituels funéraires des immigrants dans le pays d'immigration. La deuxième section présente le cas d'une famille congolaise dont les rituels funéraires n'ont pas été reconnus par le gouvernement canadien suite aux décès du couple parental de Canadiens naturalisés et grands-parents de petits-enfants nés au Canada. Le visa de résidence temporaire a été refusé deux fois à un membre de la famille qui devait arriver en provenance du Congo afin de compléter une étape importante du processus funéraire. Ce refus nous conduit à nous interroger sur la signification du sens de l'appartenance au Canada des survivants des défunts vivant au Canada. La troisième section décrit la transgression des rituels funéraires selon la culture des disparus et le sentiment de mépris de leur citoyenneté qui s'empare alors des membres de la famille vivant au Canada. La dernière section suggère l'idée d'une nécessité d'extension de la notion de citoyenneté qui tient compte des rituels funéraires des immigrants.

\section{Les rituels funéraires des immigrants}

Cette section présente brièvement les études portant sur la mort sur la terre d'accueil, puis sur les rituels funéraires en Afrique subsaharienne et chez les $\mathrm{Nande}^{2}$ en particulier et enfin, le contexte de la mort du couple d'immigrants congolais Kavira et Kitsongo.

Les recherches relatives à la mort des migrants dans les pays d'immigration traitent de cette question sous divers angles (Rachédi, Leduc et Montgomery, 2010). Blake (2007) traite des droits humains des morts, le traitement des morts en Afrique dans une perspective historique est abordé par Lee et Vaughan (2008). Berthod (2006) réfléchit sur l'équivoque identitaire au moment de la mort des immigrants portoricains aux États-Unis, tandis que Caulier (1997) pose la question de la bonne mort, c'est-à-dire celle qui répond aux rites funéraires des immigrants à Montréal. Pour Reimers (1999), les rituels funéraires des immigrants vivant en Suède constituent des actions symboliques de communication de leur identité ethnique. Toujours en Suède, Jonker (1996) montre que les musulmans sont confrontés à de nombreuses difficultés dans leur recherche, à des manières innovantes de prendre en charge les rituels de la mort dans cet État. Cette réalité des rituels mortuaires des immigrants a été également analysée dans le cas des États-Unis par Myer (1993), de même que leurs difficultés à élaborer de manière satisfaisante un processus de deuil (Gardner, 2002).

Plusieurs travaux soulignent le fait que nombre de gouvernements de pays d'immigration n'ont pas pris de mesures suffisantes afin de répondre aux impératifs des cultes funéraires des migrants malgré leur présence sur ces territoires depuis plusieurs décennies (Fall et Dimé, 2011 ; Aggoun, 2003 ; Petit, 2002).

2 Les Nande constituent un groupe ethnique de langue bantou installé en République Démocratique du Congo et en Ouganda. 
D'autres études documentent la mise en place dans les pays européens d'éléments institutionnels qui assurent la reconnaissance publique des musulmans et notamment de leurs rituels funéraires (Koeng, 2007). Aux États-Unis, Amava (2007) a montré comment des non-Américains, latino-américains, accèdent à une citoyenneté posthume grâce à leur mort sur les champs de bataille en Iraq et comment les rituels funéraires accompagnent le processus de naturalisation. Enfin, le double engagement des immigrants vis-à-vis à la fois de leur terre $d^{\prime}$ accueil et de celle de leur pays d'origine à travers leurs pratiques mortuaires est signalé par Zirh (2012).

Questionnant la signification de la mort chez les musulmans en France, notamment à travers la question du rapatriement des défunts, Chaib (2000) montre que les musulmans vivant en France pensent que les cimetières français ne répondent pas aux normes de leur culture funéraire. Le rapatriement de la dépouille mortelle dans le pays d'origine symbolise pour ces populations l'impératif du lien d'appartenance à leur pays d'origine. Pour sa part, Petit (2002) observe les migrants manjak originaires du Sénégal. Elle constate que la question de la mort des immigrants d'Afrique subsaharienne ouvre la porte à trois enjeux majeurs : tout d'abord, le choix du lieu de sépulture en pays hôte ou dans celui d'origine, l'accomplissement de rites ancrés dans des représentations collectives qui touchent à la mort et à l'affiliation, et enfin le coût financier de la prise en charge des rituels funéraires. Attias-Donfut et Wolff (2005), dans une de leur recherche portant sur le lieu d'enterrement des personnes nées hors de France, montrent que les femmes font, moins que les hommes, le choix du pays d'origine comme lieu de leur enterrement, que plus on est arrivé jeune en France plus on a tendance à vouloir être enterré dans ce pays d'installation et qu'enfin, les musulmans souhaitent davantage que les autres groupes être inhumés dans leur pays d'origine.

Les travaux relatifs aux célébrations mortuaires en Afrique subsaharienne montrent que celles-ci sont particulières aux groupes de cette région. Nwoye (2005) analyse les rituels funéraires africains en tant que système inventé par les communautés afin de les aider à surmonter efficacement les blessures psychologiques et la souffrance causée par la mort des êtres chers. Ces rituels funéraires symbolisent leurs relations profondes à leurs cultures et leur attachement à la personne décédée (Dlukulu, 2010). De manière plus spécifique, Hutchings (2007) traite des rites funéraires, Maloka (1995) des traditions mortuaires des Basotho, tandis que Manyedi, Koen et Greeff (2003) présentent les rituels des peuples du Botswana. Malgré la diversité culturelle du continent, la mort reste dans les cultures africaines, essentiellement une affaire communautaire traduisant le sens de la solidarité et les liens étroits entre les membres de la famille. Participer aux funérailles par la visite de la tombe du défunt est le passeport qu'on accorde au défunt afin qu'il puisse quitter en paix le monde des vivants et vivre en sérénité dans le monde des ancêtres (Dlukulu, 2010). Les visites des membres de la famille se doublent d'une dimension thérapeutique et de mémorialisation. Les visites de la famille sur la tombe du défunt permettent des processus de réconciliation. En aplanissant les conflits familiaux, elles permettent d'envisager l'avenir collectif sous un angle nouveau. Les visites sur la tombe fixent la mémoire des funérailles dans l'esprit des membres de la famille. Elles attachent le lieu de l'enterrement à la mémoire familiale (Fall et Dimé, 2011 ; Nwoye, 2005 ; Dlukulu, 2010). 
Les rituels mortuaires ne sont donc pas des événements détachés de la vie des individus, ils répondent à une logique qui les dépasse (Maloka, 1995). Ces pratiques rituelles sont, par nature, des activités régulées par des communautés politiques, c'est-à-dire des interactions entre les individus et les institutions du pays. Aussi, les pratiques politiques à l'égard des rituels funéraires des immigrés permettent d'évaluer la portée de la citoyenneté des immigrants. Selon Wikan (1988) qui compare des communautés musulmanes originaires d'Égypte et de Bali vivant en Angleterre, la culture constitue la variable la plus significative dans les rituels funéraires des immigrants, confirmant ce que Irish, Lundquist et Nelsen (1993) appellent la variation ethnique dans les rituels mortuaires fondée sur la culture.

Nunez et Wheeler (2012) rappellent qu'il n'est pas suffisant d'analyser les rituels funéraires des immigrants sous le seul angle du choix du lieu d'enterrement du défunt et ils proposent d'aller au-delà de ce premier niveau d'analyse. De notre point de vue, la relation entre les cérémonies funéraires de l'immigrant et le contenu de leur citoyenneté en pays d'accueil doivent prendre en compte la complexité des rituels funéraires qui englobent non seulement le lieu d'enterrement, mais aussi d'autres aspects du deuil tel que les rituels à accomplir après I'inhumation en terre d'accueil. Des études récentes sur les rites funéraires des immigrants (Fall et Dimé, 2011 ; Rachédi, Leduc et Montgomery, 2010), portant sur l'intervention lorsqu'arrive la mort chez les immigrants, n'évoquent pas ce niveau d'analyse. Pourtant, les pratiques culturelles des rituels mortuaires en relation avec la citoyenneté ne devraient laisser personne indifférent, car c'est par la mort qu'on médiatise l'ordre de la culture (Volant, 2001) et c'est la sépulture qui créé la culture (Volant, cité par Bussières, 2007) et son expression doit être considérée comme un ingrédient significatif de la citoyenneté.

Dans une analyse de l'idée de la reconnaissance et de la justice sociale, Matsumoto (2013) montre que sans reconnaissance culturelle il ne peut y avoir de justice sociale. Ce à quoi nous rajoutons, qu'il ne peut y avoir non plus de citoyenneté comme Honneth (2000) et Fraser (2005) l'argumentent. La question de la reconnaissance dans le champ de l'identité culturelle des personnes, notamment par le biais des rituels funéraires des groupes sociaux, est déterminante si I'on se réfère à Taylor (1994). Il s'agit d'une question de justice sociale si I'on se place dans la perspective développée par Rawls dans sa théorie de la justice (1971). Malgré ce qui semble être un contresens pour Rawls (1991) qui est un défenseur du droit individuel et non collectif, dans une société pluraliste, les cultures des groupes sociaux doivent être reconnues pour assurer une justice sociale. Dans un contexte du multiculturalisme, la reconnaissance de la culture des groupes ethniques contribue à la promotion de la justice sociale (Kymlicka, 1989) et elle suscite une réflexion sérieuse sur la citoyenneté multiculturelle qui fait appel à la dimension culturelle de l'idéal démocratique. Ceci conduit à une plus large intégration des rites funéraires des immigrants dans les politiques nationales (Park, 2010). Pour certains chercheurs, comme Kymlicka (1989) et Matsumoto (2013), les seules dimensions classiques de la citoyenneté politique, civile et sociale se révèlent insuffisantes dans le contexte du pluralisme culturel, car l'immigrant a le droit de conserver son identité d'origine, qui est ouverte aux apports du pays d'accueil. Ne pas reconnaître cette identité, c'est dévaloriser l'immigrant ou lui refuser ce qu'il a de plus fondamental. 
Chez les Nande, le décès d'un membre de la famille est une occasion symbolique et forte permettant de manifester l'unité familiale, de réaffirmer son appartenance à la communauté et sa loyauté à l'égard des valeurs ancestrales (Wasakundi, 1985 ; Kakiranyi, 1998). À cette fin, les célébrations funéraires, en plus de leurs fonctions d'accompagnement de la personne décédée dans l'au-delà, doivent montrer que la personne est morte conformément aux prescriptions " erihola ndenke ", c'est-à-dire que tous les membres de la famille ont pris soin de s'occuper de l'ensemble des étapes du deuil. Ceci s'oppose au mal mourir, " erihola navi ", terme exprimant le fait que tous les rituels funéraires n'ont pas été respectés ou qu'ils ont été mal exécutés. Le rituel d'accompagnement du défunt dans son passage du monde des êtres vivants à celui des ancêtres ne s'opère pas selon la volonté des survivants, mais selon les conditions établies par la communauté, c'est-à-dire une structure englobant l'individu et la famille. II $y$ a donc des étapes formelles à suivre afin d'assurer une harmonie relationnelle entre le défunt et les membres de la famille et d'affirmer son appartenance à la communauté.

Les cérémonies funéraires chez les Nande s'articulent autour de trois phases dès l'instant que la mort est constatée : I'annonce de la mort du défunt, la procédure couvrant sa mise en tombe et les opérations entourant la fin des rituels funèbres (Wasakundi, 1985 ; Kakiranyi, 1998). L'étape de I'annonce de la mort consiste à trouver une explication plausible à la mort, c'est-à-dire l'élément qui l'a causée. Cette explication doit être acceptable par les membres de la famille. II faut ajouter que tout le monde n'est pas apte à annoncer la mort. Cette tâche revient au plus âgé de la famille qui, selon la croyance, possède les qualités essentielles, en particulier la sagesse, pour annoncer la tragédie qui s'abat sur la famille (Bergmans, 1971).

Puis, arrive l'étape de la préparation du corps du défunt afin qu'il entre rapidement dans le monde des ancêtres. Avant la colonisation, les enfants n'assistaient pas à la mise en tombe du corps pour leur éviter le traumatisme émotif que cela pouvait entraîner. Après l'enterrement du corps, arrive le moment du retrait du deuil, c'est-à-dire la reconnaissance que la vie doit continuer malgré la douleur. Cette étape rappelle aux survivants que la guérison n'est possible que lorsque toutes les cérémonies funéraires se sont déroulées correctement (Kakiranyi, 1998). Au cours de cette phase, les membres immédiats de la famille du défunt se rasent les cheveux, un arbre est planté sur la tombe et quelques animaux sont sacrifiés pour que le mal causé par la mort soit expié. Durant les semaines suivantes, les membres de la famille résidant dans différentes parties du pays affluent afin de venir présenter leurs condoléances à la famille éprouvée et visiter la tombe du défunt (Wasakundi, 1985 ; Kakiranyi, 1998). Ces visites familiales sur le lieu de l'enterrement s'ancrent dans le processus de validation de l'unité familiale et l'ancestralisation du défunt. Elles constituent une marque éminente de la sacralité qui féconde la relation entre le mort et les vivants. Si ces étapes ne respectent pas les normes prescrites par la communauté, l'esprit du défunt ne pourra pas quitter le monde, car la famille aura refusé au défunt la possibilité de reposer en paix (Wasakundi, 1988). 


\section{Le contenu de la citoyenneté à l'épreuve de la transgression des rituels funéraires des immigrants}

L'exemple des décès de Kavira et Kitsongo survenus au Canada va nous permettre d'identifier les entorses à la citoyenneté canadienne des immigrants dans le contexte de la mort en terre d'accueil. Mère et père de treize enfants, ils sont arrivés au Canada comme résidents permanents. Ils y ont vécu quelques années avant d'y mourir et d'y être enterrés. Kavira décéda la première, causant l'émoi de sa famille parce qu'elle arrivait d'un pays dévasté par la guerre et qu'elle l'avait fui pour un monde meilleur. Leurs enfants sont canadiens de naissance ou le sont devenus par naturalisation. Les enfants naturalisés s'y plaisaient et croyaient, jusqu'à la mort de leurs parents, jouir des avantages que la citoyenneté canadienne est censée offrir. Malheureusement, la mort de ces derniers a révélé les iniquités dissimulées à l'intérieur des pratiques politiques. Ceux nés avec la nationalité canadienne, contents d'avoir finalement accolés des sentiments positifs concrets sur les charges émotives qu'apportent les interrelations entre grands-parents et petits-enfants, ont brutalement découvert que leur citoyenneté obtenue à la naissance, cachait des trous béants qui la rendait peu attrayante.

Kitsongo, le mari de Kavira, observait les pratiques sociales des Canadiens lorsqu'est survenue la mort de son épouse en terre d'accueil. La communauté congolaise de Vancouver et d'autres régions du Canada rendirent hommage à son épouse comme il se doit culturellement. Après une rapide discussion entre ses enfants résidant au Canada et ceux restés au Congo, la volonté de Kavira d'être enterrée au Canada fut exhaussée. Elle fut conduite au cimetière de Gethsémani dans la banlieue de Vancouver. Pour Kitsongo, la visite de la tombe de son épouse par certains membres de sa famille restés au Congo serait automatique. Malheureusement, Kitsongo mourut un mois après son épouse, après cinquante ans de mariage, et fut inhumé à ses côtés dans le même cimetière. Quelques semaines avant sa mort, Kitsongo avait émis le vœu de réaliser une partie du rituel obituaire qui consiste, dans la culture Nande, à planter sur la terre familiale un arbre symbolisant la tombe du défunt enterrée loin de sa terre natale (Wasakundi, 1981). Par la suite, la dernière étape consisterait à faire venir au Canada, un de ses fils vivant au Congo afin qu'il puisse au nom de tous les membres de la famille s'incliner devant la tombe de sa mère Kavira. La visite de la tombe par un membre de la famille venu du Congo devait assurer le passage de Kavira du monde des vivants à celui des ancêtres et clore ainsi le processus de guérison des survivants. Malheureusement, Kitsongo mourut avant d'avoir voyagé et répondu à cette exigence culturelle.

II faut garder à l'esprit que les États ont un rôle de premier plan dans le traitement des restes humains parce qu'ils les contrôlent et les assignent à des espaces délimités (Esquerre et Truc, 2011). Même si certains ne peuvent pas établir une relation étroite entre les rituels mortuaires et les politiques nationales, il est indéniable que l'exclusion de la culture mortuaire du contenu de la citoyenneté n'est pas accidentelle, mais qu'elle est plutôt une construction sociopolitique, c'est-à-dire que certaines cultures sont exclues par des politiques gouvernementales. Un des indicateurs de cette exclusion culturelle est le refus $\mathrm{d}^{\prime}$ accorder un visa de résidence temporaire à une personne qui souhaite venir s'incliner sur la tombe de ses parents morts au Canada. Le refus du visa de 
résidence temporaire est dans ce cas précis une pratique d'exclusion fondée sur le rejet de la culture de l'immigrant. Pourtant, dans toutes les sociétés humaines, les survivants du défunt ont des obligations envers le mort et la société (Valentine et Woodthorpe, 2013). Ils ont une dette sociale qui implique d'exprimer leurs émotions selon certains codes et pratiques transmis de génération en génération. Le deuil occasionne chez les survivants des souffrances profondes qui ne peuvent être apaisées que si les rituels qui accompagnent le processus de guérison ne font pas l'objet de transgressions (Shange, 2009 ; Opperman et Novello, 2006 ; Drenth, Herbst et Strydom, 2010).

L'attitude de l'officier de l'immigration pour justifier le refus d'accorder le visa de résidence temporaire à la personne invitée au Canada rend encore plus problématique cette pratique d'exclusion culturelle. En effet, il était clairement indiqué sur la demande de visa que la personne qui était invitée par ses frères et sœurs vivant au Canada, venait s'incliner sur les tombes de Kavira et Kitsongo afin de poursuivre la réalisation des cérémonies funéraires. Les deux fois où le visa a été sollicité, l'officier de l'immigration a indiqué au demandeur : "Vous ne prouvez pas que vous avez suffisamment de moyens financiers pour pouvoir retourner dans votre pays ". Pour l'officier de l'immigration, le statut social et économique de la personne invitée est davantage déterminant que le respect de la dimension multiculturelle (Kymlicha, 1995) des enfants canadiens de Kavira et Kitsongo vivant au Canada. Le manque de reconnaissance sociale et culturelle à l'égard d'un individu ou d'un groupe a été interprété comme une atteinte à la fois aux droits et à la dignité humaine par certains philosophes contemporains (Raymond, 2011 ; Honneth, 2000 ; Ricœur, 2004 ; Renault, 2004). De plus, le Canada se targue d'être un État promouvant le multiculturalisme et des droits de la personne quelles que soient les cultures ou les religions des individus (Kymlicka, 2006). L'article 15 du Pacte international relatif aux droits économiques, sociaux et culturels en son alinéa 2 affirme que les États participant au présent pacte prendront des mesures en vue d'assurer le maintien, le développement et la diffusion de la science et de la culture des individus et des groupes (Addo, 2010). Dans la lettre d'invitation adressée à l'ambassade du Canada afin de solliciter le visa de résidence temporaire de la personne qui devait venir du Congo, il était expressément indiqué que la venue de cette personne constituait une des exigences des rituels funéraires chez les Nande. Pour l'officier de l'immigration, qui représente le gouvernement canadien et applique ses politiques d'immigration, cette partie de la culture des Nande vivant au Canada n'a pas été prise en compte. Ne pas permettre à la descendance canadienne de Kavira et Kitsongo de compléter une étape importante des rituels funéraires, c'est les condamner non seulement à ne pas entreprendre leur processus de guérison, mais aussi les pousser à questionner la véritable portée de leur citoyenneté canadienne.

La construction de la citoyenneté culturelle élargit la lecture traditionnelle de la citoyenneté. Pour Wang (2013), I'affirmation de la culture des groupes ethniques procède du droit des minorités et va au-delà des dimensions traditionnelles politique, sociale et politique de la citoyenneté. Cette approche renvoie à la distinction entre immigrants autorisés, mais non reconnus établie par Sassen (2002). À la lumière des faits évoqués précédemment, Kavira, Kitsongo et leurs enfants sont des immigrants autorisés, mais non reconnus parce que leur culture est niée et que par conséquent leur citoyenneté est bafouée. Parce 
que les cérémonies funéraires sont étroitement liées aux politiques nationales, ceux et celles qui sont perçus comme appartenant à la communauté politique dans laquelle ils vivent jouissent d'un deuil digne de leurs droits culturels. Par contre, ceux et celles qui y sont considérés comme des étrangers voient leurs cultures funéraires déniées. C'est dire que la lecture de la citoyenneté des survivants de Kavira et Kitsongo, sous l'angle des rituels funéraires des Nande, permet de déterminer quelle mort a de l'importance politique et quelle autre en est dépourvue (Pool, 2012 ; Seery, 1996 ; Stevens, 2010). En niant l'importance politique des morts de Kavira et Kitsongo, I'État prouvait à leur descendance que leur citoyenneté canadienne est de seconde zone.

\section{Conclusion}

Conscient du fait que les représentations de la mort et le processus de guérison du traumatisme que la mort entraine au sein des familles, les gouvernements ouverts à la migration internationale, devraient davantage intégrer dans leurs politiques nationales des éléments qui répondent aux rituels funéraires des migrants en tant qu'impératifs de justice sociale et culturelle. La réflexion suggère que si le traitement politique des rites funéraires des immigrants est irrespectueux des pratiques mortuaires des pays d'origine des immigrants, le sentiment positif d'appartenir à leur pays d'accueil et le lien d'attachement à leur nouvelle citoyenneté peuvent être anéantis. Cette réflexion sur le processus de deuil, lorsque survient la mort d'un immigrant en terre d'accueil, indique qu'il faut une analyse plus poussée sur cette question qui semble être un terrain potentiellement fertile pour démasquer en partie les véritables enjeux de la pratique de la citoyenneté culturelle. Cette discussion suggère aussi que le traitement politique de la culture funéraire des immigrants qui reconnaît aux immigrants le statut de citoyens pluri-identitaires, accroît en eux le sens d'une pleine citoyenneté.

Par ailleurs, la discussion permet d'attirer l'attention sur le fait que les seuls aspects traditionnels de la citoyenneté ne répondent plus aux réalités d'une citoyenneté moderne constituée de multiples constellations (Baubock, 2010). Mieux encore, il faut répondre à l'inévitable extension de la citoyenneté comme le propose Joppke (2010) vers, entre autres, les rituels funéraires. En d'autres termes, il faut permettre aux migrants d'enterrer les membres de leurs familles dans la dignité et le respect de leurs cultures. Enfin, reconnaître les rituels funéraires des groupes ethniques c'est répondre à ce que Walzer (1985) appelle la reconnaissance d'une des sphères de la justice sociale dans le contexte du pluralisme et de la poursuite de l'égalité des citoyens. 


\section{Références bibliographiques}

Addo Michael (2010) The practice of United Nations human rights treaty bodies in the reconciliation of cultural diversity with universal respect for human rights, Human Rights Quarterly, 32 (3), pp. 601-664.

Aggoun Atmane (2003) Immigration, grands-parents algériens et mémoire : entre la transmission et I'oubli, L'homme et la société, 147-148, pp. 191-207.

Amaya Hector (2007) Latino immigrants in the American discourses of citizenship and nationalism during the Iraqi war, Critical Discourse Studies, 4 (3), pp. 237-256.

Attias-Donfut Claudine et François-Charles Wolff (2005) Le lieu d'enterrement des personnes nées hors de France, Population, 5 (60), pp. 813-816, [en ligne]. URL : http://www.cairn.info/resume.php?ID_ARTICLE=POPU_505_0813

Baubock Rainer (2010) Studying citizenship constellations, Journal of Ethnic and Migration Studies, 36 (5), pp. 847-859.

Bergmans Lieven (1971) Les Wanande. Croyances et pratiques traditionnelles, T. 2, Butembo, Editions ABB, $134 \mathrm{p}$.

Bernstein Jay M. (2005) Suffering injustice: Misrecognition as moral injury in critical theory, International Journal of Philosophical Studies, 13 (3), pp. 303-324.

Berthod Marc Antoine (2006) Expérience migratoire et identité dans la mort transnationale : les défunts portoricains rapatriés de NewYork, Revue Canadienne des Études Latino-américaines et Caraïbes, 61 (31), pp. 145-168.

Blake Janet (2007) Beyond death: the treatment of indigenous human rights - a human rights perspective, Islam and Christian-Muslim Relations, 18 (3), pp. 367-375.

Blauner Robert (1966) Death and social structure, Psychiatry, 29, pp. 378-394.

Bussières Luc (2007) Rites funèbres et sciences humaines : synthèse et hypothèses. Nouvelles perspectives en sciences sociales, Revue internationale de systémique complexe et d'études relationnelles, 3 (1), pp. 61-139, [en ligne] consulté le 23/03/2013. URL : http://www.erudit.org/revue/npss/2007/v3/ n1/602466ar.pdf

Caulier Brigitte (1997) Pour une bonne mort : les confréries et la mort à Montréal (XVIle-XIXe siècles), Frontières, 5 (1), pp. 14-18.

Chaïb Yassine (2000) L'émigré et la mort, Aix-en-Provence, CIDIM-Edisud, 255 p.

Dlukulu Puseletso Masebolao (2010) Black urban widows: Their experiences of and coping with bereavement in a transitional society, Thèse de doctorat non publiée, Pretoria : South Africa, University of Pretoria.

Drenth Cornelia, Herbst Alida G. and Strydom Herman (2010) A complicated grief intervention model, Health SA Gesondheid, 15 (1), pp. 1-8, [online]. URL: http:// www.hsag.co.za/index.php/HSAG/article/view/415/480

Esquerre Arnaud et Truc Gérôme (2011) Les morts, leurs lieux et leurs liens, Raisons politiques, 41 (1), pp. 5-11.

Fall Khadiatoulah et Dime Mamadou Ndongo (2011) La mort musulmane en contexte d'immigration et d'islam minoritaire. Enjeux religieux, culturels, identitaires et espaces de négociation, Québec, Presses de I'Université Laval, 228 p. 
Fraser Nancy (2011) Qu'est-ce que la justice sociale ? Reconnaissance et redistribution, Paris, La Découverte, $178 \mathrm{p}$.

Gardner Katy (2002) Death of a migrant: Transnational death rituals and gender among British Sylhetis, Global Networks, 2 (3), pp. 191-204.

Honneth Axel (2000) La lutte pour la reconnaissance, Paris, Cerf, 194 p.

Hutchings Anne (2007) Ritual cleansing, incense and the tree of life - observations on some indigenous plant usage in traditional Zulu and Xhosa purification and burial rites, Alternation, 14 (2), pp. 189-218.

Irish Donald, Lundquist Kathleen and Nelsen Vivian (1993) Ethnic variations in dying, death and grief: Diversity in universality, Washington, DC, Taylor \& Francis, $226 \mathrm{p}$.

Jonker Gerdien (1996) The knife's edge: Muslim burial in the diaspora, Mortality, 1 (1), pp. 27-43.

Joppke Christian (2010) The inevitable lightening of citizenship, European Journal of Sociology/Archives européennes de sociologie, 51 (1), pp. 9-32.

Kadrouch-Outmany Khadija (2013) Burial practices and desires among Muslims in the Netherlands: A matter of belonging, Canadian Journal of Netherlandic Studies/Revue cannadienne d'études néerlandaises, 34 (1), pp. 107-128.

Kakiranyi Kule (1998) Le munande (Yira) et ses traditions, Butembo, Éditions G.I.B.

Koenig Mathias (2007) Europeanising the governance of religious diversity: An institutionalist account of Muslim struggles for public recognition, Journal of Ethnic and Migration Studies, 23 (6), pp. 911-932.

Kymlicka Will (1995) Multicultural citizenship, London, Oxford University Press, $280 \mathrm{p}$.

Kymlicka Will (1989) Liberalism, community and culture, Oxford, Oxford University Press, 280 p.

Lee Rebekah and Vaughan Megan (2008) Death and dying in the history of Africa since 1800, Journal of African History, 49, pp. 341-359.

Lestage Françoise (2012) Éditorial : La mort en migration. Revue européenne des migrations internationales, 28 (3), [en ligne] consulté le 23/03/2013. URL : http:// remi.revues.org/5992

Maloka Tshidiso (1995) Basotho and the experience of death, dying and mourning in the South African mine compounds, 1890-1940, Cahiers d'études africaines, 1890-1940 (Centre for African Studies 1890-1940, 38 (149), pp. 17-40, [online] last checked on 17/01/2013. URL : http://www.jstor.org/stable/4392848.

Manyedi Eva, Koen Cur and Greeff Cur (2003) Experiences of widowhood and beliefs about the mourning process of the Batswana people, Health SA Gesondheid, 8 (4), pp. 69-87.

Matsumoto Masakazu (2013) Political theorizing and policy implications: The case of a Rawlsian approach to multicultural education, International Journal of Multicultural Education, 15 (1), [online] last checked on 17/11/2012. URL: http:// ijme-journal.org/index.php/ijme/article/view/559/pdf 
Metcalf Peter and Huntington Richard (1991) Celebrations of death: The anthropology of mortuary ritual, Cambridge, Cambridge University Press, 236 p.

Myer Richard (1993) Ethnicity and the American cemetery, Bowling Green, KY, Bowling Green State University Popular Press, 239 p.

Nunez Laura and Wheeler Brittany (2012) Chronicles of death out of place: Management of migrant death in Johannesburg, African Studies, 71 (2), pp. 212-233.

Nwoye Augustine (2005) Memory healing processes and community intervention in grief work in Africa, Australia and New Zealand Journal of family Therapy, 26 (3), pp. 147-154.

Opperman B. and Novello A. (2006) The generation of hypotheses with regard to the influence of context on complicated grief, South African Journal of Psychology, 36 (2), pp. 374-390.

Park Chang Wong (2010) Cultural blending in Korean death rites: New interpretative approaches, London/New York, Continuum, 227 p.

Petit Agathe (2002) La mort au loin. Les pratiques funéraires des migrants africains en France, Thèse d'ethnologie et d'anthropologie sociale, Marseille, EHSSS, ss la dir. d'André Mary.

Pool Heather (2012) The Politics of mourning: The Triangle Fire and political belonging, Polity, 44 (2), pp. 182-211.

Rachédi Lilyane, Leduc Véronique et Montgomery Catherine (2010) Accompagner les familles immigrantes endeuillées : mieux comprendre pour mieux intervenir, [en ligne] consulté le 17/11/2012, URL : http://www.csssdelamontagne.qc.ca/ fileadmin/csss_dlm/Publications/Publications_CRF/bulletin_1-low.pdf

Rawls John (1971) A theory of justice, Cambridge, MA, The Belknap Press of Harvard University Press, 607 p.

Raymond Charles (2011) Le retour des sentiments moraux dans la théorie de la reconnaissance, [en ligne] consulté le 10/02/2013. URL : https://halshs.archivesouvertes.fr/halshs-00670677/document

Reimers Eva (1999) Death and identity: Graves and funerals as cultural communication, Mortality, 4 (2), pp. 147-166.

Renault Emmanuel (2004) L'expérience de l'injustice. Reconnaissance et clinique de l'injustice, Paris, La Découverte, 412 p.

Ricœur Paul (2004) Parcours de la reconnaissance, Paris, Stock, 448 p.

Sassen Saskia (2002) The Repositioning of citizenship: Emergent subjects and spaces for politics, Berkeley Journal of Sociology, 4, [online] last checked on 11/01/2013. URL: http://transnationalism.uchicago.edu/RepositioningCitizenship. pdf

Seery John (1996) Political theory for mortals: Shades of justice, images of death, Ithaca, NY, Cornell University Press, 248 p.

Shange Lindiwe (2009) Bereaved Employees in Organisations: Managers and workers Co-Responsibility, Unpublished DoctoralThesis, Zululand : South Africa, University of Zululand. 
Stevens Jacqueline (2010) States without nations: Citizenship for mortals, New York, Columbia University Press, 384 p.

Taylor Charles (1994) Multiculturalisme, différence et démocratie, Paris, Aubier, $378 \mathrm{p}$.

Valentine Christine and Woodthorpe Kate (2013) Death \& society: A global introduction, London and Thousand Oaks, Sage, 240 p.

Van den Breemer, Rosemarie and Marcel Maussen (2012) On the Viability of State-Church Models: Muslims Burial and Mosque Building in France and the Netherlands, Journal of Immigrant \& Refugee Studies, 10 (3), pp 279-298, [online]. URL: http://www.tandfonline.com/doi/abs/10.1080/15562948.2012.6930 37\#preview

Volant Éric (2001) La religion et la mort, in Jean Marc Larouche et Guy Ménard Éds., L'étude de la religion au Québec. Bilan et prospective, Québec, Les Presses de I'Université Laval/Corporation canadienne des sciences religieuses, pp. 323-342.

Walzer Michael (1985) Spheres of justice: A defense of pluralism and equality, New York, Basic Books, 345 p.

Wang Li Jung (2013) Towards cultural citizenship? Cultural rights and cultural policy in Taiwan, Citizenship Studies, 17 (1), pp. 92-110.

Wasakundi Athanase (1981) Dieu Nyamuhanga chez les Nande du Zaïre. Essai d'une christologie africaine dans ses rapports avec l'évangélisation, Thèse de doctorat non publiée, Lyon, Faculté de Théologie de Lyon.

Wikan Unni (1988) Bereavement and loss in two Muslim communities: Egypt and Bali compared, Social Science \& Medicine, 27 (5), pp. 451-460.

Zirh Bessim Can (2012) Following the dead beyond the "nation": A map for transnational Alevi funerary routes from Europe to Turkey, Ethnic and Racial Studies, 35 (10), pp. 1758-1774. 